\title{
28 Research Suare \\ Categorisation of Sit-To-Stand Motion Pattern. Human vs Automated Quantitative Assessments.
}

Mirko Job ( $\square$ mirko.job.1991@gmail.com )

Universita degli Studi di Genova https://orcid.org/0000-0002-0991-420X

\section{Simone Battista}

Universita degli Studi di Genova

Ronny Stanzani

Universita degli Studi di Genova

Alessio Signori

Universita degli Studi di Genova

Marco Testa

Universita degli Studi di Genova

\section{Research}

Keywords: Movement Pattern, Sit-To-Stand, Reliability, Movement Recognition

Posted Date: July 24th, 2020

DOI: https://doi.org/10.21203/rs.3.rs-47639/v1

License: (a) (i) This work is licensed under a Creative Commons Attribution 4.0 International License. Read Full License 


\section{Abstract}

BACKGROUND: The Sit-to-Stand (STS) test is widely used in clinical practice as an indicator of lower-limb functionality decline, especially for older adults. Hitherto, due to its high variability, there is no standard approach for categorising the STS motion pattern, and the vision-based evaluation remains the most reliable method to evaluate people' performance. This paper presents a comparative analysis between visual assessments and an automated-software approach for the categorisation of STS, relying on registrations from a force plate.

METHODS: A group of 5 participants ( $30 \pm 6$ years) took part in 2 different sessions of visual inspections on 200 STS movements randomly extracted from a dataset of 742 acquisitions under self-paced and controlled speed conditions. Assessors were asked to identify three specific STS events from the Ground Reaction Force, simultaneously with the software analysis: the start of the trunk movement (Initiation), the beginning of the stable upright stance (Standing) and the sitting movement (Sitting). The Test-Retest Reliability between first and second visual evaluations was compared with the Inter-Rater Agreement between visual and software assessments, as indexes of human and software performance, respectively.

RESULTS: No statistical differences between methods were found for the identification of the Initiation and the Sitting events at self-paced speed and for only the Sitting event at controlled speed. The estimated significant values of maximum discrepancy between visual and automated assessments were $0.200 \mathrm{~s}[0,039 ; 0.361]$ in unconstrained conditions and $0,340 \mathrm{~s}[0,014 ; 0,666]$ for standardised movements.

CONCLUSIONS: The software assessments displayed an overall good agreement against visual evaluations of the Ground Reaction Force, relying, at the same time, on objective measures. In this sense, the proposed approach can provide robust and consistent data in the field of Big Data analytics, augmenting the performance of artificial intelligence methods for Human Activity Recognition tasks.

\section{Background}

Performance-based tests are important clinical tools used to identify functional decline in older adults and patients with either neurological or motor impairments [1]. Among them, the Sit-to-Stand test (STS) is strictly correlated with functional capacity and with independence in the activities of daily living [2-9]. Furthermore, its simplicity allows it to be performed both in clinical and in-home environments across a broad range of patients' functional conditions $[1,5]$. For these reasons, the STS movement is widely used in clinical research and practice, either as a single test [10-12] or as part of evaluation scales like the Short Physical Performance Battery [13]. Diversely from gait, a standardised categorisation for the STS motion pattern has yet to be established, due to its intrinsic variability. Furthermore, the lack of consensus in the definitions and measurement methodologies increases the uncertainty even in the definition of those events that normally are generally recognisable. For instance, the lifting of the thighs from the chair is defined across the literature as the peak instant of the horizontal [14] and vertical components [15] of the Ground Reaction Force (GRF), the time of maximum anterior head movement [16] or through a sensor-equipped chair [17].

In an attempt to describe a general model of the movement, Nuzik [18] divided the STS into two phases through the analysis of camera recordings, i.e. the flexion phase, consisting of the forward flexion of trunk and hip, and 
the extension phase, characterised by the lifting from the chair to the extension of knees, hips, and ankles. In later years, a finer sub-categorisation was introduced by Schenkman and colleagues [19]. They identified other two phases, with the use of a motion capture system, i.e. a "momentum transfer phase" between the flexion phase and the extension phase, when the inertia is transferred from the trunk flexion to the upper body, and a "stabilisation phase" when the hip is completely extended, and all the movements associated with stabilisation from rising are completed. Differently, Etnyre and colleagues analysed the kinetics of the STS under different conditions to recognise eleven invariably occurring events in the GRF, i.e. six in the vertical direction, three in the fore-aft direction, and two in the medial-lateral direction [20].

In general, despite the large amount of data obtained from a wide variety of sensors and technologies, it is still not possible to identify a univocal approach for a standardised definition of the STS motion pattern. Kinematic parameters proved to be strongly affected by the high within-between individuals' variability, limiting the performance of automated techniques to the recognition of dynamic transitions and static positions. On the contrary, kinetic variables permit a finer discretisation of the movement, but they still depend too much on visual evaluations to obtain reliable results $[20,21]$.

According to the previous statements, to establish an objective method to describe commonly occurring events in STS is essential to assure quantitative and repetitive measures to be used as a reference in clinical practice and research.

\section{Methods}

\section{OBJECTIVE}

This study aims at evaluating the performance of a new algorithm able to automatically recognise specific events in the STS movement from GRF profiles and at comparing its results with the reliability displayed by human visual assessments. The routine implementation is described in detail in an additional document (see Additional File 1).

\section{DEFINITION OF THE DATASETS}

We evaluated the performance of the proposed method on two Human Activity Recognition datasets (HAR1 and HAR2), collected at the REHELab (University Campus of Savona, Via Magliotto 2, 17100, Savona, Italy). Both HAR1 and HAR2 represent a series of sequential movements collected from a convenience sample of healthy young adults (Table 1). The inclusion criteria for eligible subjects were: good health, absence of musculoskeletal or neurological disorders and, ability to easily rise from a chair. Each participant had to sign an informed consent. 
Table 1

\begin{tabular}{|c|c|c|}
\hline HAR1 & & HAR2 \\
\hline 19 & $\mathrm{~N}^{\circ}$ Subjects & 20 \\
\hline $26 \pm 3$ & Age [years] & $27 \pm 4$ \\
\hline [9/10] & Gender [M/F] & [11/9] \\
\hline $62,7 \pm 10,3$ & Weight [kg] & $67,3 \pm 15,0$ \\
\hline $171 \pm 9$ & Height [m] & $172 \pm 9$ \\
\hline
\end{tabular}

Participants performed the STS repetitions on a force plate (Kistler Winterthur, Switzerland). During the execution of the movement, a custom-made chair equipped with an electronic switch was able to record the time instants of rising (Seat Off) and sitting (Seat On). In both datasets, participants had to execute two different tasks:

- To perform 10 repetitions of a single STS transition at self-paced speed (SP);

- To perform 10 repetitions of a single STS transition at controlled speed (CT) with duration marked by a repetitive 4 seconds acoustic feedback, composed by a succession of 3 tones and a pause.

The different strategies of motor control involved in the two tasks outlined two typical profiles of the Ground Reaction Force (GRF). According to the definition of STS events given by Etnyre and colleagues [20], in SP trials, an initial deflection from the baseline was observed (Initiation). After reaching the lowest level in the force recording (Peak-counter), the GRF raise to a global maximum (Peak) and subsequently levelled to a normal postural sway (Standing). Diversely, CT trials were characterised by a more gradual increase in the GRF following the progressive inclination of the trunk and the raising movement from the chair. Examples of force profiles for both SP and CT trials are displayed in Fig. 1.

Hence, the STS movement pattern was categorised in 4 sequential phases (Fig. 2):

- the Rest phase (RES), identified as the initial sitting position;

- the Trunk Leaning phase (TLN), starting from the Initiation event to the Seat Off instant;

- the Raising phase (RAI), delimited from the Seat Off to the Standing event as the first instant of steady postural sway;

- the Standing phase (STA), characterised by a stable upright position until the beginning of the sitting movement.

The two datasets are the result of two consecutive studies. Hence, some minor modification in the execution protocol of the exercise must be disclosed and taken into account. These changes are due to the progressive methodological improvement of the general study, which relies on previous empirical evidence to reduce possible future bias. The summary of the protocol differences between the two datasets is reported in Table 2 . 
Table 2

Protocol differences between HAR1 and HAR2 datasets

\begin{tabular}{|ll|}
\hline HAR1 & HAR2 \\
\hline $\begin{array}{l}\text { In SP trials some participants } \\
\text { tended to start the movement } \\
\text { too early not allowing the } \\
\text { registration of an appropriate } \\
\text { RES phase. }\end{array}$ & $\begin{array}{l}\text { In SP trials participants had to wait } 3 \text { seconds from the start of the } \\
\text { acquisition before starting the movement. }\end{array}$ \\
$\begin{array}{ll}\text { In SP trials some participants } \\
\text { tended to sit-down without } \\
\text { having reached a sufficient } \\
\text { stable STA phase. }\end{array}$ & $\begin{array}{l}\text { In SP trials participants had to wait } 3 \text { seconds in STA phase to reach } \\
\text { balance stability. }\end{array}$ \\
$\begin{array}{l}\text { In CT trials participants } \\
\text { considered the Stand-to-Sit } \\
\text { transition as a single } \\
\text { returning phase, starting from } \\
\text { the beginning of the Sitting } \\
\text { event until reaching the RES } \\
\text { phase. }\end{array}$ & $\begin{array}{l}\text { In CT trials participants were asked to control the descending movement, } \\
\text { identifying two returning phases: a "Sitting" phase, starting from the } \\
\text { beginning of the sitting movement until registration of the "seat-on" signal; } \\
\text { a Trunk Raising phase, where subjects raise the trunk until reaching the } \\
\text { the Stand-to-Sit transition. }\end{array}$ \\
\hline
\end{tabular}

\section{EVALUATION OF THE SOFTWARE PERFORMANCE}

In the lack of a proper gold standard approach, visual inspection remains the most reliable method to recognise STS events and phases on the base of GRF values [20]. Hence, simultaneously with the software analysis, five participants aged $(30 \pm 6)$ years visually identified the beginning of the trunk movement (Initiation event) and the limits of the stable stance (Standing and Sitting events) from the force profiles of 200 STS sequences. The ages of the assessors are reported in the table below (Table 3) together with their professional and academic background.

Table 3

Assessors' description.

\begin{tabular}{|lll|}
\hline AGE & $\begin{array}{l}\text { PROFESSIONAL } \\
\text { BACKGROUND }\end{array}$ & ACADEMIC LEVEL \\
\hline 29 & Bioengineer & MSc/PhD student \\
\hline 27 & Physicist & MSc/PhD student \\
40 & Physiotherapist & PhD \\
\hline 25 & Bioengineer & MSc/Research Fellow \\
\hline 30 & Psychologist & MSc/PhD student \\
\hline
\end{tabular}

As a measure of reliability, assessments were repeated in 2 distinct sessions, separated in time by a minimum interval of 1 hour. STS sequences were drawn randomly from a total pool of 742 acquisitions (HAR1 + HAR2) and were maintained across the measurement trials. This information was not made explicit to avoid possible learning effects. Among the 200 pooled sequences, 100 referred to SP trials, and 100 referred to CT trials. Before the first session, subjects were briefly trained to recognise the onset of each event on five force profiles accordingly to the definitions given by Etnyre and colleagues [20], and an explanatory summary was always 
available in the form of MATLAB live script throughout all the measurements. All assessors were physiotherapists, bioengineers and PhD candidates with clinical experience in physiotherapy and expertise in movement analysis.

\section{DATA ANALYSIS}

Test-Retest reliability was considered as a quality index of the human measure, and it was compared with the performance of the software evaluated with the Inter-Rater reliability against participants' assessments in the first trial. For both analyses, the absolute agreement was measured using a non-parametric version of BlandAltman statistics [22], as a consequence of the violation of the normality assumption of data. Normal distributions were tested using the Kolmogorov-Smirnov test and, furtherly investigated using the skewness and kurtosis indexes. To correct the analysis for possible outliers not directly connected to the evaluation of the assessors or the performance of the software, data observations that fell outside 1.5 interquartile ranges above the upper quartile (75 percentile) or below the lower quartile (25 percentile), were visually inspected. In particular, wrong identifications resulting from an erroneous right mouse click, early movements or a malfunction of the electronic switch were eliminated. We calculated the systematic bias and the Limits of Agreement as the median of the absolute differences between assessments and the respective 2.5-th and 97,4-th percentile scores. The upper Limits of Agreement (ULoA) represented the maximum estimated error between the measures. The 95\% Confidence Intervals (Cl) of the above-specified parameters were also calculated using a percentile bootstrap method based on $10 \mathrm{k}$ samples [23]. The percentile method was chosen for its conservative nature, as it tends to produce wider $\mathrm{Cl}$ less sensitive to population value and sample size [24]. To compare the maximum errors made between the Test-Retest trials and the Human-Software evaluations, we used a twotailed two-sample t-test [25], exploring the significant differences between measures. Every ULoAs and respective $\mathrm{Cl}$ were approximated to normal distributions characterised by mean values $\mu_{i}$ and standard deviation $\sigma_{i}$ [26], calculated as:

$$
\begin{aligned}
\mu_{i}=U L o A_{i} \\
\sigma_{i}=\left\{\begin{array}{lll}
\frac{\left|C I_{\text {up }}-(U L o A)_{i}\right|}{1,96} \cdot \sqrt{N} & \text { if } & \left|C I_{\text {up }}-(U L o A)_{i}\right|>\left|C I_{\text {low }}-(U L O A)_{i}\right| \\
\frac{\left|C I_{\text {low }}-(U L O A)_{i}\right|}{1,96} \cdot \sqrt{N} & \text { if } & \left|C I_{\text {low }}-(U L O A)_{i}\right|>\left|C I_{\text {up }}-(U L O A)_{i}\right|
\end{array}\right.
\end{aligned}
$$

The choice of the lower or higher confidence limit is guided by the need to calculate the largest standard error to obtain a more conservative approximation. The statistical analysis was stratified by considering the different STS events separately, dividing the results obtained in SP and CT trials. Bland-Altman statistics was implemented in MATLAB as a modified version of the BlandA/tman.m function developed by Ran Klein from the Department of Nuclear Medicine of the Ottawa Hospital [27]. The two-tailed two-sample t-test was executed with the online "Comparison of means calculator" tool from Medcalc Statistical Software [28].

\section{Results}

The Bland-Altman plots relating to the identification of the Initiation, Standing and Sitting events are shown respectively in Fig. 3, Fig. 4 and Fig. 5 and the summary of the descriptive statistics of bias and the ULoAs (CI) are reported in Table 4. 
Table 4

Descriptive statistics for Bias and ULoA for every comparison. ( $\left.{ }^{\star}\right) \mathrm{p}<0,05$ for not overlapping Cl.

\begin{tabular}{|c|c|c|c|c|c|c|c|c|c|c|c|c|}
\hline \multirow{4}{*}{ Events } & \multicolumn{6}{|c|}{ SP trials } & \multicolumn{6}{|c|}{ CT trials } \\
\hline & \multicolumn{3}{|c|}{ Test-Retest } & \multicolumn{3}{|c|}{ Human-Software } & \multicolumn{3}{|c|}{ Test-Retest } & \multicolumn{3}{|c|}{ Human-Software } \\
\hline & Bias & ULoA & $\mathrm{N}$ & Bias & ULoA & $\mathrm{N}$ & Bias & ULoA & $\mathrm{N}$ & Bias & ULoA & $\mathrm{N}$ \\
\hline & {$[\mathrm{s}]$} & [s] & & [s] & {$[\mathrm{s}]$} & & {$[s]$} & {$[\mathrm{s}]$} & & {$[\mathrm{s}]$} & {$[s]$} & \\
\hline \multirow[t]{3}{*}{ Initiation } & 0,06 & 0,36 & \multirow[t]{3}{*}{492} & 0,14 & 0,46 & \multirow[t]{3}{*}{490} & 0,14 & 0,62 & \multirow[t]{3}{*}{483} & 0,18 & 0,96 & \multirow[t]{3}{*}{493} \\
\hline & {$[0,05$} & {$[0,30$} & & {$[0,12$} & {$[0,39$} & & {$[0,12$} & {$[0,54$} & & {$[0,16$} & {$[0,84$} & \\
\hline & $0,06]$ & $0,46]$ & & $0,15]$ & $0,58]$ & & $0,16]$ & $0,84]$ & & $0,20]$ & $1,20]$ & \\
\hline \multirow[t]{3}{*}{ Standing } & 0,10 & 0,62 & \multirow[t]{3}{*}{488} & 0,30 & 0,82 & \multirow[t]{3}{*}{495} & 0,16 & 1,30 & \multirow[t]{3}{*}{484} & 0,44 & 1,60 & \multirow[t]{3}{*}{493} \\
\hline & {$[0,08$} & {$[0,48$} & & {$[0,28$} & {$[0,74$} & & {$[0,16$} & {$[1,10$} & & \multirow{2}{*}{$\begin{array}{l}{[0,40} \\
0,48]\end{array}$} & {$[1,40$} & \\
\hline & $0,10]$ & $0,74]$ & & $0,32]$ & $0,86]$ & & $0,20]$ & $1,70]$ & & & $1,70]$ & \\
\hline \multirow[t]{5}{*}{ Sitting } & 0,08 & 0,42 & \multirow[t]{3}{*}{497} & 0,20 & 0,48 & \multirow[t]{3}{*}{494} & 0,14 & 0,69 & \multirow[t]{3}{*}{491} & 0,20 & 0,81 & \multirow[t]{3}{*}{490} \\
\hline & {$[0,06$} & {$[0,34$} & & {$[0,18$} & {$[0,46$} & & {$[0,12$} & {$[0,60$} & & {$[0,18$} & {$[0,66$} & \\
\hline & $0,08]$ & $0,47]$ & & $0,22]$ & $0,60]$ & & $0,14]$ & $0,86]$ & & $0,22]$ & $0,86]$ & \\
\hline & & * & & & & & * & & & & & \\
\hline & & * & & & & & & & & & & \\
\hline
\end{tabular}

In both SP and CT trials, for all STS events, participants showed significantly lower identification bias in the repeated assessments, compared to the difference displayed against the measurements performed by the software. Moreover, the Test-Retest Reliability in the identification of the STS events significantly decreases in CT trials with a consequent increase in the value of the systematic error.

The result of the two-tailed two-sample t-test are summarised in Table 5. 
Table 5

Two-tailed two-sample t-test results. Differences with p-values $<0,05\left(^{*}\right)$ were considered statistically significant.

\begin{tabular}{|lllllll|}
\hline \multicolumn{5}{|c|}{ SP trials } & \multicolumn{5}{c|}{ CT trials } \\
\hline & Initiation & Standing & Sitting & Initiation & Standing & Sitting \\
\hline Difference [s] & 0,100 & 0,200 & 0,060 & 0,340 & 0,300 & 0,120 \\
\hline $95 \% \mathrm{Cl}[\mathrm{s}]$ & $-0,056$ & 0,039 & $-0,084$ & 0,014 & 0,017 & $-0,107$ \\
& 0,256 & 0,361 & 0,204 & 0,666 & 0,583 & 0,347 \\
\hline t-statistic & 1,255 & 2,440 & 0,816 & 2,045 & 2,079 & 1,037 \\
\hline df & 980 & 981 & 989 & 974 & 975 & 979 \\
\hline p-value & 0,209 & $0,015\left(^{*}\right)$ & 0,414 & $0,041\left(^{*}\right)$ & $0,038\left(^{*}\right)$ & 0,300 \\
\hline
\end{tabular}

Significant differences in ULoAs between Test-Retest evaluation and Human-Software assessments were found in the Standing event identification for both SP $(0,200 s[0,039 ; 0,361], p>0,05)$ and CT $(0,300 s[0,017 ; 0,583], p$ $>0,05)$ trials. A significant difference $(p>0,05)$ of $0,340 s[0,014 ; 0,666]$ was also found in the Initiation event identification in CT trials.

\section{Discussion}

This work aimed at evaluating the performance of a new automated approach for the recognition of clinically relevant events in the STS and at comparing its performance to the human visual assessment. The results obtained offer a double contribution in prospective researches since not only we quantified the discrepancy between the two methods, but we also compared it against the maximum error made in repeated visual measures. Despite the significant lower systematic bias in repeated evaluations, the comparison between visual assessments and the proposed approach showed similar values of maximum absolute error.

More specifically no statistical differences were found in the identification of the Initiation and the Sitting events in SP trials and in the identification of only the Sitting event in CT trials. The worsening of the observed agreement during CT movements was generally in line with our expectation, as ULoAs values could be affected by uncertainties due to the kinetic modifications resulting from the standardisation of the movement. For instance, the initial GRF deflection effect is highly dependent on each individual's movement strategy [20] and could be reduced by the lower quantity of momentum produced under constrained speed, complicating the identification of the Initiation event.

Another important consideration highlights the intrinsic subjectiveness of human evaluations [29]. One could consider the slightest oscillation either as an extension of a contiguous static phase or as the limit of a movement transition. Moreover, visual assessments can vary across repeated evaluation and differ in individuals, depending on their professional experience [30, 31].

Nonetheless, with a maximum estimated discrepancy of $0,200 s[0,039 ; 0,361]$ and $0,340 s[0,014 ; 0,666]$ respectively for normal and standardised speed, the proposed method may not be suitable for evaluations on a 
single patient, where the expertise of health professionals plays a key role in the diagnosis process, often requiring a high level of abstraction [29]. It is also true that a great quantity of data collected through sensor systems is inherently noisy, and for this reason, its analysis should consider and handle some degree of uncertainty [32]. In this context, the presented algorithm can be used as a solid basis for artificial intelligence methods to provide accurate, faster, and scalable results in the field of big data analytics with interesting applications in Human Activity Recognition tasks [33, 34].

This is the first study that aims at comparing human and automated assessments in the identification of a complex motion pattern in the STS movement relying on data collected through a force plate. Previous works [35-39] evaluated the performances of various algorithms developed for the identification of Sit-to-Stand and Stand-to-Sit postural transitions using data acquired from inertial sensors. In particular, a recent paper of Atrsaei and colleagues [40] validated the accuracy of a new routine based on a single device against visual assessments on-camera recordings of STS movements, obtaining levels of agreement above $94 \%$, in terms of positive predictive values and sensitivity. As a direct comparison with the present study, the use of inertial sensors is usually preferable since they can be also applied in non-clinical environments [41]. However, their measurements are strongly influenced by the inter- and intra-individual variability of the movement [20,42], limiting the recognition of the STS motion pattern to the simple discrimination of static and dynamic phases. Conversely, our choice to use a force plate has some doubtless limitations in terms of costs and portability but the strong advantage of providing easier interpretable results, on which it is possible to identify clinically significant movement events and phases. Under this point of view, supported by the recent advances in the field of machine learning, it is possible to consider this data as a valid ground-truth reference to train specific IMUbased approaches in a finer recognition of the STS. This transition could allow the development of accessible, wearable rehabilitation tools, combining the discriminating power of gold-standard instruments with the limited dimensions and costs of inertial sensors.

The results highlighted in this work should be appraised in consideration of some limitations. The standardisation of posture at the beginning of the trials and the homogeneity of the sampled population in HAR1 and HAR2 could have restricted the applicability of the presented evidence. Both the setting of the starting position and the individuals' characteristics influence the STS motion strategy and consequently, the value of the vertical GRF and the successful outcome of the movement $[20,43,44]$. Hence, the presented algorithm must be tested across a different set of initial conditions (e.g. different uses of the arms during the rising, different fatigue condition of the subject, etc.) and different population groups to explore its real value for clinical applications. Moreover, the limited sample size and the heterogeneous professional background of the assessors could have impacted the final estimate of the visual measurements. Further evaluations performed by a larger sample of qualified health professionals could provide a more precise picture of the reliability of the proposed method.

\section{Conclusion}

The presented algorithm displayed an overall good agreement against visual assessments in the identification of significant STS events from values of vertical Ground Reaction Force. By providing objective and fast measures, this automated method can be applied for the analysis of a large amount of data to identify specific 
trends and patterns in patients' movement. Further studies should be undertaken to validate its accuracy across different types of patients' conditions.

\section{Abbreviations}

Cl

Confidence Intervals; GRF:Ground Reaction Force; HAR:Human Activity Recognition; RAl:Raising; RES:Resting; SP:Self-Paced speed; STA:Standing; STS:Sit-To-Stand; TLN:Trunk Leaning; ULoA:Upper Limits of Agreement.

\section{Declarations}

\section{ETHICS APPROVAL AND CONSENT TO PARTICIPATE}

All participants which composed the analysed datasets had to sign an informed consent.

\section{CONSENT FOR PUBLICATION}

Not applicable.

\section{AVAILABILITY OF DATA AND MATERIALS}

The software used to support the conclusions of this article is partially described within the Additional file 1 and a complete implementation is available at https://zenodo.org/record/3956138\#.XxgQgigzZPY [45]. The datasets used and analysed during the current study are available from the corresponding author on reasonable request.

\section{COMPETING INTERESTS}

The authors declare that they have no competing interests.

\section{FUNDING}

This work was not supported by any specific funding. Ronny Stanzani is supported by Liguria Region European Social Fund Operational Program (POR-FSE) 2014-2020.

\section{AUTHORS' CONTRIBUTIONS}

MT conceived and directed the study and participated in the drafting process and in the interpretation of data. MJ defined the design of the study, developed the analysis and the experimental software, participated in the interpretation of data and drafted the manuscript. SB and AS helped in the drafting process, defined the statistical analysis and participated in the interpretation of data. RS participated in the development of the 
software, helped in the drafting process and participated in the interpretation of data. All authors have seen

and approved the final version of the manuscript.

\section{ACKNOWLEDGEMENTS}

Not applicable.

\section{References}

1. Soubra R, Chkeir A, Novella J-L. A Systematic Review of Thirty-One Assessment Tests to Evaluate Mobility in Older Adults. Biomed Res Int [Internet]. 2019 [cited 2019 Dec 18];2019. Available from: https://www.ncbi.nlm.nih.gov/pmc/articles/PMC6610744/.

2. Bohannon RW. Measurement of Sit-to-Stand Among Older Adults. Topics in Geriatric Rehabilitation. 2012;28:11.

3. Dall PM, Kerr A. Frequency of the sit to stand task: An observational study of free-living adults. Appl Ergon. 2010;41:58-61.

4. Gross MM, Stevenson PJ, Charette SL, Pyka G, Marcus R. Effect of muscle strength and movement speed on the biomechanics of rising from a chair in healthy elderly and young women. Gait Posture. 1998;8:17585.

5. Hellmers S, Fudickar S, Lau S, Elgert L, Diekmann R, Bauer JM, et al. Measurement of the Chair Rise Performance of Older People Based on Force Plates and IMUs. Sensors. 2019;19:1370.

6. http://doi.acm.10.1145/3154862.3154882

Hellmers S, Steen E-E, Dasenbrock L, Heinks A, Bauer JM, Fudickar S, et al. Towards a Minimized Unsupervised Technical Assessment of Physical Performance in Domestic Environments. Proceedings of the 11th EAI International Conference on Pervasive Computing Technologies for Healthcare [Internet]. New York, NY, USA: ACM; 2017 [cited 2019 Dec 11]. p. 207-216. Available from:

http://doi.acm.org/10.1145/3154862.3154882.

7. Inkster LM, Eng JJ. Postural control during a sit-to-stand task in individuals with mild Parkinson's disease. Exp Brain Res. 2004;154:33-8.

8. Jeyasurya J, Van der Loos HFM, Hodgson A, Croft EA. Comparison of seat, waist, and arm sit-to-stand assistance modalities in elderly population. J Rehabil Res Dev. 2013;50:835-44.

9. Munton JS, Ellis MI, Chamberlain MA, Wright V. An investigation into the problems of easy chairs used by the arthritic and the elderly. Rheumatol Rehabil. 1981;20:164-73.

10. Bohannon RW. Reference values for the five-repetition sit-to-stand test: a descriptive meta-analysis of data from elders. Percept Mot Skills. 2006;103:215-22.

11. Csuka M, McCarty DJ. Simple method for measurement of lower extremity muscle strength. Am J Med. 1985;78:77-81.

12. Jones CJ, Rikli RE, Beam WC. A 30-s chair-stand test as a measure of lower body strength in communityresiding older adults. Res Q Exerc Sport. 1999;70:113-9. 
13. Guralnik JM, Simonsick EM, Ferrucci L, Glynn RJ, Berkman LF, Blazer DG, et al. A short physical performance battery assessing lower extremity function: association with self-reported disability and prediction of mortality and nursing home admission. J Gerontol. 1994;49:M85-94.

14. Doorenbosch CA, Harlaar J, Roebroeck ME, Lankhorst GJ. Two strategies of transferring from sit-to-stand; the activation of monoarticular and biarticular muscles. J Biomech. 1994;27:1299-307.

15. Kaya BK, Krebs DE, Riley PO. Dynamic stability in elders: momentum control in locomotor ADL. J Gerontol A Biol Sci Med Sci. 1998;53:M126-34.

16. Alexander NB, Schultz AB, Warwick DN. Rising from a chair: effects of age and functional ability on performance biomechanics. J Gerontol. 1991;46:M91-8.

17. van Lummel RC, Ainsworth E, Hausdorff JM, Lindemann U, Beek PJ, van Dieën JH. Validation of seat-off and seat-on in repeated sit-to-stand movements using a single-body-fixed sensor. Physiol Meas. 2012;33:1855-67.

18. Nuzik S, Lamb R, VanSant A, Hirt S. Sit-to-stand movement pattern. A kinematic study. Phys Ther. 1986;66:1708-13.

19. Schenkman M, Berger RA, Riley PO, Mann RW, Hodge WA. Whole-body movements during rising to standing from sitting. Phys Ther. 1990;70:638-51.

20. Etnyre B, Thomas DQ. Event standardization of sit-to-stand movements. Phys Ther. 2007;87:1651-66.

21. Piano L, Geri T, Testa M. Raising and stabilization phase of the sit-to-stand movement better discriminate healthy elderly adults from young subjects: a pilot cross-sectional study. Arch Physiother [Internet]. 2020 [cited $2020 \mathrm{Jul}$ 12];10. Available from: https://www.ncbi.nlm.nih.gov/pmc/articles/PMC7161000/.

22. Bland JM, Altman DG. Statistical methods for assessing agreement between two methods of clinical measurement. Lancet. 1986;1:307-10.

23. Davison AC, Hinkley DV. Bootstrap Methods and their Application. 1 edizione. Cambridge University Press; 1997.

24. Jung K, Lee J, Gupta V, Cho G. Comparison of Bootstrap Confidence Interval Methods for GSCA Using a Monte Carlo Simulation. Front Psychol [Internet]. 2019 [cited 2020 Jul 5];10. Available from: https://www.ncbi.nlm.nih.gov/pmc/articles/PMC6797821/.

25. Altman DG. Practical Statistics for Medical Research. CRC Press; 1990.

26. Chapter 6. Choosing effect measures and computing estimates of effect | Cochrane Training [Internet]. [cited $2020 \mathrm{Jul}$ 20]. Available from: https://training.cochrane.org/handbook/current/chapter-06.

27. Bland-Altman. and Correlation Plot [Internet]. [cited 2020 Jul 5]. Available from: https://it.mathworks.com/matlabcentral/fileexchange/45049-bland-altman-and-correlation-plot.

28. Schoonjans F. MedCalc's Comparison of means calculator [Internet]. MedCalc. [cited 2020 Jul 5]. Available from: https://www.medcalc.org/calc/comparison_of_means.php.

29. O'Connor AB. Clinical Instruction \& Evaluation: A Teaching Resource. Jones \& Bartlett Publishers; 2014.

30. Jaarsma T, Jarodzka H, Nap M, van Merriënboer JJG, Boshuizen HPA. Expertise in clinical pathology: combining the visual and cognitive perspective. Adv in Health Sci Educ. 2015;20:1089-106.

31. Persky AM, Robinson JD. Moving from Novice to Expertise and Its Implications for Instruction. AJPE. 2017;81:6065. 
32. Hariri RH, Fredericks EM, Bowers KM. Uncertainty in big data analytics: survey, opportunities, and challenges. Journal of Big Data. 2019;6:44.

33. Li T, Chen J, Hu C, Ma Y, Wu Z, Wan W, et al. Automatic Timed Up-and-Go Sub-Task Segmentation for Parkinson's Disease Patients Using Video-Based Activity Classification. IEEE Trans Neural Syst Rehabil Eng. 2018;26:2189-99.

34. Greene BR, Doheny EP, Kenny RA, Caulfield B. Classification of frailty and falls history using a combination of sensor-based mobility assessments. Physiol Meas. 2014;35:2053-66.

35. Najafi B, Aminian K, Paraschiv-lonescu A, Loew F, Büla CJ, Robert P. Ambulatory system for human motion analysis using a kinematic sensor: monitoring of daily physical activity in the elderly. IEEE Trans Biomed Eng. 2003;50:711-23.

36. Godfrey A, Bourke AK, Olaighin GM, van de Ven P, Nelson J. Activity classification using a single chest mounted tri-axial accelerometer. Med Eng Phys. 2011;33:1127-35.

37. Salarian A, Russmann H, Vingerhoets FJG, Burkhard PR, Aminian K. Ambulatory monitoring of physical activities in patients with Parkinson's disease. IEEE Trans Biomed Eng. 2007;54:2296-9.

38. 10.3389/fneur.2018.00652/full

Pham MH, Warmerdam E, Elshehabi M, Schlenstedt C, Bergeest L-M, Heller M, et al. Validation of a Lower Back "Wearable"-Based Sit-to-Stand and Stand-to-Sit Algorithm for Patients With Parkinson's Disease and Older Adults in a Home-Like Environment. Front Neurol [Internet]. 2018 [cited 2020 Jul 13];9. Available from: https://www.frontiersin.org/articles/10.3389/fneur.2018.00652/full.

39. Rodríguez-Martín D, Samà A, Pérez-López C, Català A. Identification of sit-to-stand and stand-to-sit transitions using a single inertial sensor. Stud Health Technol Inform. 2012;177:113-7.

40. Atrsaei A, Dadashi F, Hansen C, Warmerdam E, Mariani B, Maetzler W, et al. Postural transitions detection and characterization in healthy and patient populations using a single waist sensor. J Neuroeng Rehabil. 2020;17:70.

41. Job M, Dottor A, Viceconti A, Testa M. Ecological Gait as a Fall Indicator in Older Adults: A Systematic Review. Gerontologist Oxford Academic. 2020;60:e395-412.

42. Kristiansen M, Rasmussen GHF, Sloth ME, Voigt M. Inter- and intra-individual variability in the kinematics of the back squat. Hum Mov Sci. 2019;67:102510.

43. Noh H-J, Kim C-Y, Kim H-D, Kim S-W. Changes in Muscle Activation and Ground Reaction Force of the Lower Limbs According to Foot Placement During Sit-to-Stand Training in Stroke Patients. Am J Phys Med Rehabil. 2020;99:330-7.

44. Shen S, Abe T, Tsuji T, Fujii K, Ma J, Okura T. The relationship between ground reaction force in sit-to-stand movement and lower extremity function in community-dwelling Japanese older adults using long-term care insurance services. J Phys Ther Sci. 2017;29:1561-6.

45. 10.5281/zenodo.3956138

Job M. Sit-To-Stand-Categorisation. Zenodo; 2020. http://doi.org/10.5281/zenodo.3956138.

46. ADDITIONAL FILES.

47. Additional. File 1 - Software Implementation for the recognition of the Sit-To-Stand (STS) events.

48. This document (.pdf) contains the detailed description of the automated software with commented code. 
Figures

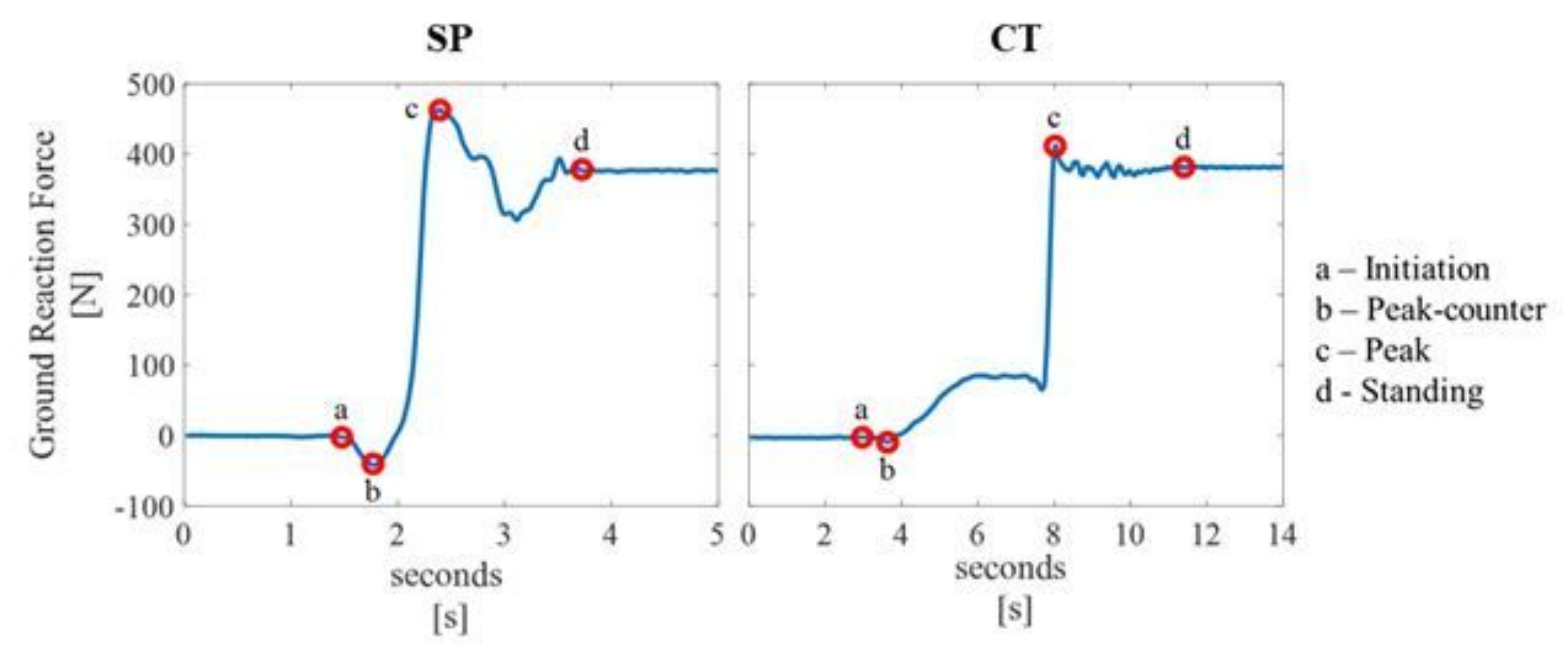

Figure 1

GRF profiles of SP and CT trials with identification of STS events.

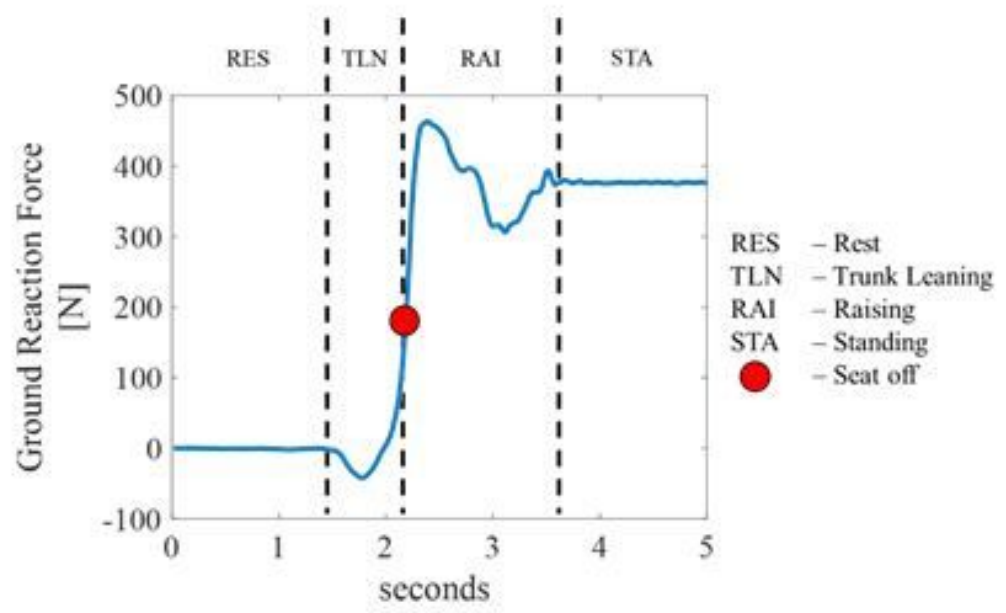

[s]

Figure 2

Categorisation of STS phases 

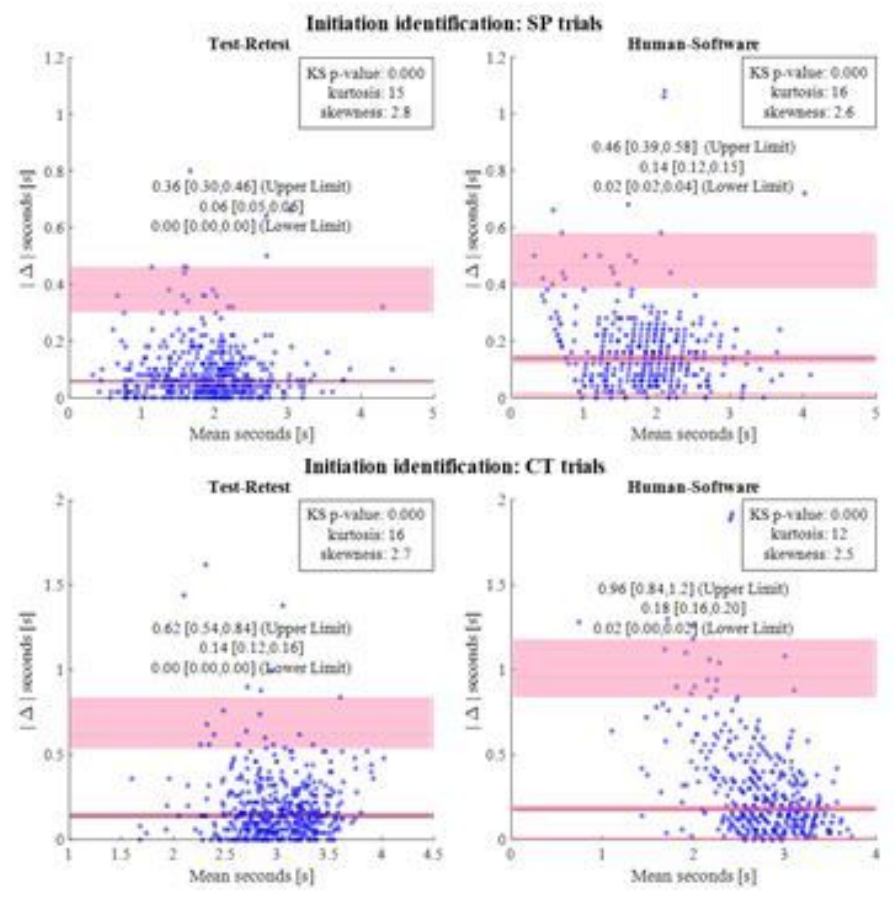

Figure 3

Bland-Altman plots depicting the Test-Retest Reliability (left) and the Inter-Rater Reliability (right) of the identification of the Initiation event in SP trials (upper plots) and CT trials (lower plots).
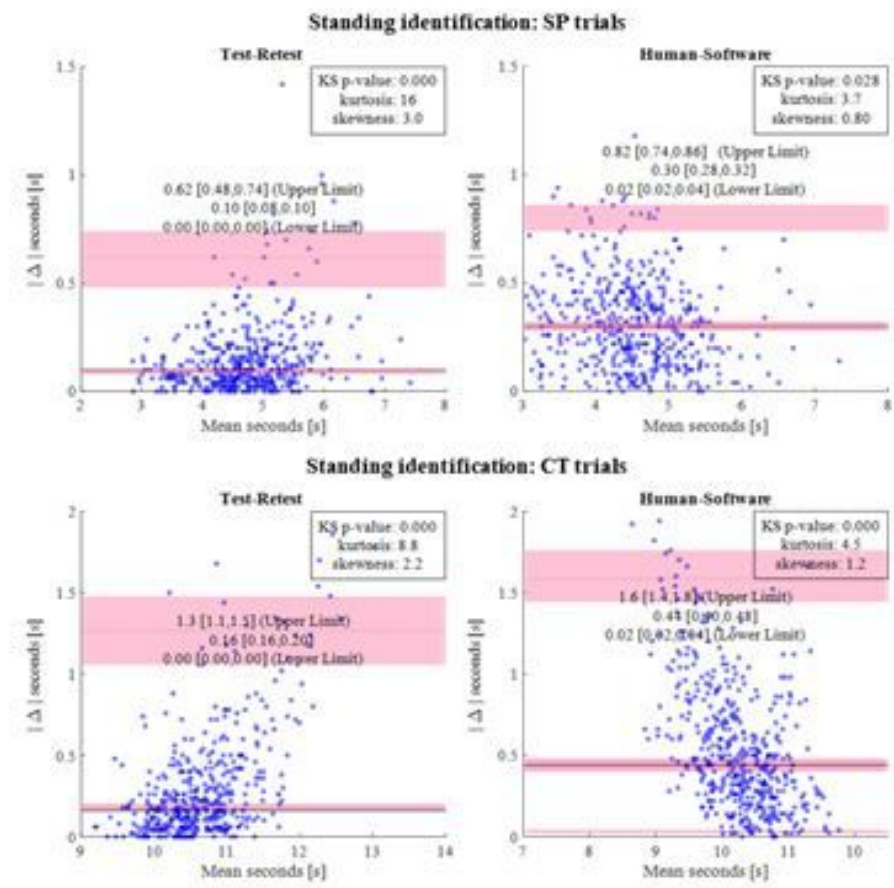

\section{Figure 4}

Bland-Altman plots depicting the Test-Retest reliability (left) and the Inter-Rater reliability (right) of the identification of the Standing event in Task A (upper plots) and Task B (lower plots). 

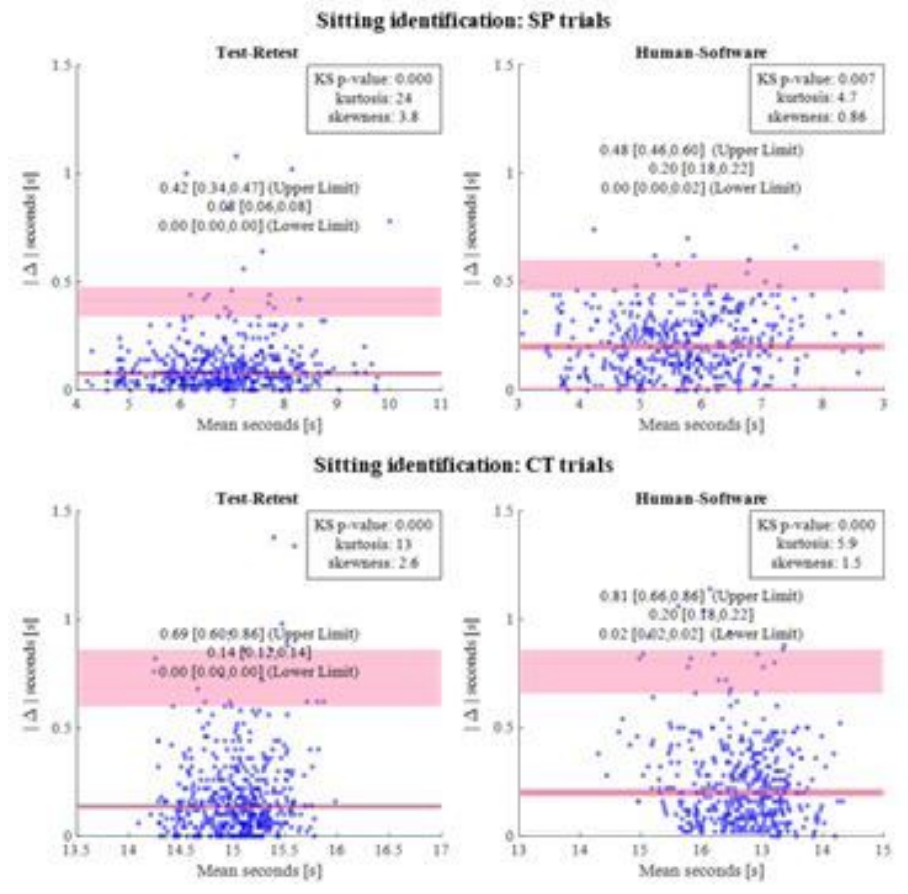

Figure 5

Bland-Altman plots depicting the Test-Retest Reliability (left) and the Inter-Rater Reliability (right) of the identification of the Standing event in SP trials (upper plots) and CT trials (lower plots).

\section{Supplementary Files}

This is a list of supplementary files associated with this preprint. Click to download.

- AdditionalFile1.pdf 